\title{
Mapping Sex-Specific Neurodevelopmental Alterations in Neurite Density and Morphology in a Rat Genetic Model of Psychiatric Illness
}

\author{
Brian R. Barnett, ${ }^{1}$ Sue Y. Yi, ${ }^{1}$ McKenzie J. Poetzel, ${ }^{2}$ Keith Dodd, ${ }^{2}$ Nicholas A. Stowe, ${ }^{2}$ and \\ (D)John-Paul J. Yu' $1,2,3,4$
}

https://doi.org/10.1523/ENEURO.0426-20.2020

${ }^{1}$ Neuroscience Training Program, Wisconsin Institutes for Medical Research, University of Wisconsin-Madison, Madison, WI 53705, ${ }^{2}$ Department of Radiology, University of Wisconsin School of Medicine and Public Health, Madison, WI 53705, ${ }^{3}$ Department of Biomedical Engineering, College of Engineering, University of Wisconsin-Madison, Madison, WI 53706, and ${ }^{4}$ Department of Psychiatry, University of Wisconsin School of Medicine and Public Health, Madison, WI 53705

\begin{abstract}
Neurite orientation dispersion and density imaging (NODDI) is an emerging magnetic resonance (MR) diffusionweighted imaging (DWI) technique that permits non-invasive quantitative assessment of neurite density and morphology. NODDI has improved our ability to image neuronal microstructure over conventional techniques such as diffusion tensor imaging (DTI) and is particularly suited for studies of the developing brain as it can measure and characterize the dynamic changes occurring in dendrite cytoarchitecture that are critical to early brain development. Neurodevelopmental alterations to the diffusion tensor have been reported in psychiatric illness, but it remains unknown whether advanced DWI techniques such as NODDI are able to sensitively and specifically detect neurodevelopmental changes in brain microstructure beyond those provided by DTI. We show, in an extension of our previous work with a Disc1 sv $\Delta 2$ rat genetic model of psychiatric illness, the enhanced sensitivity and specificity of NODDI to identify neurodevelopmental and sex-specific changes in brain microstructure that are otherwise difficult to observe with DTI and further corroborate observed changes in brain microstructure to differences in sex-specific systems-level animal behavior. Together, these findings inform the potential application and clinical translational utility of NODDI in studies of brain microstructure in psychiatric illness throughout neurodevelopment and further, the ability of advanced DWI methods such as NODDI to examine the role of biological sex and its influence on brain microstructure in psychiatric illness.
\end{abstract}

Key words: diffusion-weighted imaging; Disc1; DTI; MRI; NODDI; rat

\section{Significance Statement}

This research presents the first demonstration of the ability of neurite orientation dispersion and density imaging (NODDI) multicompartment diffusion imaging to uncover both neurodevelopmental and sex-specific alterations in brain microstructure in psychiatric illness. We show, in a genetic Disc 1 sv $\Delta 2$ rat model, sexspecific neurodevelopmental patterns of neural microstructural change with NODDI and corresponding evidence of sex differences in behavioral endophenotypes of anxiety, cognition, and general activity. Together, our results support the potential impact and translational utility of NODDI to identify salient neurodevelopmental and sex-specific changes in brain microstructure in psychiatric illness beyond traditional morphometric and diffusion tensor approaches currently employed.

Received September 30, 2020; accepted December 28, 2020; First published January 13, 2021.

The authors declare no competing financial interests.
Author contributions: B.R.B., S.Y.Y., and J.-P.J.Y. designed research; B.R.B., S.Y.Y., M.J.P., K.D., and N.A.S. performed research; B.R.B., S.Y.Y., N.A.S., and J.-P.J.Y. analyzed data; B.R.B. and J.-P.J.Y. wrote the paper. 


\section{Introduction}

Animal models of psychiatric illness play a crucial role in furthering our understanding of the genetic, molecular, and microstructural (Gold et al., 2016; Gandal et al., 2018) features that contribute to the psychiatric disease state in numerous illnesses including autism spectrum disorder, schizophrenia, bipolar disorder, and major depressive disorder. As with other genetic variants that have been shown to confer an increased risk for psychiatric disease (Cross-Disorder Group of the Psychiatric Genomics Consortium et al., 2013), the balanced chromosomal $t(1 ; 11)(q 42.1 ; q 14.3)$ translocation of the DISC1 gene has been implicated in several psychiatric illnesses including schizophrenia (Hodgkinson et al., 2004; Callicott et al., 2005; Hamshere et al., 2005), bipolar disorder (Hodgkinson et al., 2004), autism spectrum disorder (Kilpinen et al., 2008), and major depressive disorder (Hashimoto et al., 2006) and has emerged as a key biomolecular entry point toward understanding how shared genetic perturbations underpin a broad and diverse spectrum of psychiatric illness. Buttressing the longstanding interest and import of DISC1 in neuroscience and neuropsychiatric research is a diverse repertoire of translational genetic animal models centered on the DISC1 gene (Clapcote et al., 2007; Hikida et al., 2007). In addition to these murine models, a novel rat short genetic variant model of DISC1 truncation (Disc1 sv $\Delta 2$ ) lacking exons 2-13 following targeted deletion with CRISPR/Cas9 has been generated and reported (Barnett et al., 2019). Importantly, owing to the larger brain volumes and thus greater signal-tonoise ratios that this model provides, this newly generated rat model is far more amenable to advanced magnetic resonance (MR) preclinical neuroimaging studies and crucially, can serve as a model neuroimaging system to test emerging advances in MR imaging (MRI) and to inform future clinical and translational imaging studies of neuropsychiatric illness.

While the majority of previously conducted investigations of neurodevelopmental alterations use measures of diffusion tensor imaging (DTI) and other diffusion-weighted imaging (DWI) techniques, it remains unknown whether newly developed advanced multicompartment DWI techniques such as neurite orientation dispersion and density imaging (NODDI) are able to sensitively and specifically detect neurodevelopmental changes in brain microstructure beyond those provided by conventional DWI techniques.

J.-P.J.Y. was supported by the Clinical and Translational Science Award program through the National Institutes of Health National Center for Advancing Translational Sciences Grant UL1TR002373. Additional imaging support was provided by the University of Wisconsin Carbone Cancer Center Support Grant P30CA014520 and Waisman Core Grants P30 HD003352-45 and U54 Al117924-03.

Acknowledgements: We thank the University of Wisconsin Biotechnology Center Gene and the Genome Editing and Animals Core for outstanding support; Beth Rauch for outstanding imaging support through the Small Animal Imaging Facility at the University of Wisconsin Carbone Cancer Center; and bioinformatics support from the Institute for Clinical and Translational Research (ICTR) at the University of Wisconsin-Madison.

Correspondence should be addressed to John-Paul J. Yu at jpyu@ uwhealth.org.

https://doi.org/10.1523/ENEURO.0426-20.2020

Copyright $@ 2021$ Barnett et al.

This is an open-access article distributed under the terms of the Creative Commons Attribution 4.0 International license, which permits unrestricted use, distribution and reproduction in any medium provided that the original work is properly attributed.
NODDI represents an extension of single-compartment diffusion tensor models like DTI. Whereas quantitative indices of DTI such as fractional anisotropy (FA) are able to capture neural microstructural features but are inherently non-specific, multicompartment diffusion techniques can model water diffusion across multiple compartments that enable measurement of neurite density and orientation that represent biophysically relevant features in regions with variation in synaptic density and organization.

We sought to determine the ability of NODDI to detect both neurodevelopmental and sex-specific changes in brain microstructure in a Disc1 $\mathrm{sv} \Delta 2$ rat genetic model and to corroborate changes in brain microstructure with systems-level behavioral studies. We report that male Disc 1 sv $\Delta 2$ animals demonstrate a significant decrease in orientation dispersion [orientation dispersion index (ODI)] that are matched with concomitant deficits in measures of anxiety, hyperactivity, and cognition. We found evidence of strong sex-specific differences with female Disc1 sv $\Delta 2$ animals harboring significantly higher neurite density index (NDI) and ODI than sex-matched and age-matched controls while exhibiting no significant alterations to behavioral endophenotypes of anxiety or cognition. Taken together, our findings represent the first demonstration of NODDI diffusion imaging for the identification of sex-specific neurodevelopmental changes in brain microstructure in a genetic model of psychiatric illness and supports the clinical translational utility of NODDI for the study of brain microstructure beyond traditional morphometric and diffusion tensor approaches currently employed.

\section{Materials and Methods}

\section{Subjects}

Animals were housed and cared for in an Association for Assessment and Accreditation of Laboratory Animal Care (AAALAC)-accredited facility and all animal experiments were conducted in accordance with local Institutional Animal Care and Use Committee (IACUC)-approved protocols. Outbred control male and female Sprague Dawley rats (300-325 g, Charles River) and Disc 1 sv $\Delta 2$ male and female rats (generated as described in Barnett et al., 2019) were pair housed in clear cages and were maintained under a 12/12 h light/dark cycle in humidity-controlled and temperature-controlled rooms with ad libitum access to food and water. Sprague Dawley pregnant dams were ordered from Charles River and all Sprague Dawley and Disc1 sv $\Delta 2$ male and female rats used in our data analyses were born, weaned, and matured to adulthood in the same housing facility. Animals were acclimated to housing conditions for $7 \mathrm{~d}$ before experimental manipulation. To generate the experimental Disc1 sv $\Delta 2$ animals, all Disc1 sv $\Delta 2$ male and female animals were generated from Disc1 sv $\Delta 2$ male-female homozygous pairings and subsequently genotyped to confirm genetic background (Barnett et al., 2019).

\section{Behavior analysis}

Wild-type Sprague Dawley control rats and Disc1 sv $\Delta 2$ model rats $(n=43 ; 9$ male and 10 female Disc 1 sv $\Delta 2$ rats; 
12 male and 12 female wild-type rats) at postnatal day (P) 120-P150 were tested on the elevated-plus maze, Ymaze, and open field on three consecutive days. Sample sizes for behavioral assays were calculated according to prior power analyses from co-authors' previous behavioral experiments. An hour before each day of testing, the animals were brought from their holding area to the experimental room to acclimate for $1 \mathrm{~h}$. The same nonblinded experimenter handled the animals and conducted the behavioral assays to minimize experimenter variation. Blinding to the genotype of the animals undergoing testing was not necessary, as it did not affect parameter outcomes measured in these tasks. All animals underwent the same sequence of behavioral assays on each of the three consecutive days.

The open field task determines general activity levels, gross locomotor activity, and exploration habits. Assessment took place in a square, black plastic box. The animal was placed in the arena and allowed to freely move about for $10 \mathrm{~min}$ while being recorded by an overhead camera. The footage was then analyzed by an automated tracking system for the following parameters: distance moved, velocity, and time spent in predefined zones.

The $\mathrm{Y}$-maze is a behavioral test for measuring the willingness of rodents to explore new environments. Rodents typically prefer to investigate a new arm of the maze rather than returning to one that was previously visited. Testing occurred in a Y-shaped maze with three black, opaque plastic arms at a $120^{\circ}$ angle from each other. After introduction to the center of the maze, the animal could freely explore the three arms. Over the course of multiple arm entries, the subject should show a tendency to enter a less recently visited arm. The number of arm entries and the number of triads was recorded to calculate the percentage of alternation. An entry occurred when all four limbs are within the arm.

The elevated-plus maze is used to assess anxiety-related behavior. The elevated-plus maze apparatus consisted of a plus-shaped maze elevated above the floor with two oppositely positioned closed arms, two oppositely positioned open arms, and a center area. As subjects freely explored the maze, their behavior was recorded by means of a video camera mounted above the maze and analyzed using a video tracking system. The preference for being in open arms over closed arms (expressed as either as a percentage of entries and/or a percentage of time spent in the open arms) was calculated to measure anxiety-like behavior.

\section{Imaging methodology}

Following the completion of the full battery of behavioral assays, outbred Sprague Dawley (control) rats and Disc1 sv $\Delta 2$ model rats $(n=24$; six male and female per rat group) at an average age of P135 (full range P120-P150) were brought to a surgical plane of anesthesia before sequential transcardial perfusion with ice-cold PBS and $4 \%$ paraformaldehyde (PFA). The brains were cleanly dissected from the cranial vault and imaged with a $4.7 \mathrm{~T}$ Agilent MRI system and a $3.5-\mathrm{cm}$ diameter quadrature volume RF coil. 3D multi-slice, diffusion weighted, spin echo protocols were used to acquire 10 non-diffusion weighted images $\left(b=0 \mathrm{~s} / \mathrm{mm}^{2}\right)$ and 75 diffusion-weighted images (25 non-colinear diffusion-weighting directions at $\mathrm{b}=800 \mathrm{~s} / \mathrm{mm}^{2}, 50$ non-colinear diffusion-weighting directions at $b=3500 \mathrm{~s} / \mathrm{mm}^{2}$ ). Other imaging parameters: TE/ $\mathrm{TR}=24.17 / 2000 \mathrm{~ms}, \mathrm{FOV}=30 \times 30 \mathrm{~mm}^{2}$, matrix $=192-$ $\times 192$ reconstructed to $256 \times 256$ for an isotropic voxel size of $0.25 \mathrm{~mm}$ over two signal averages. Raw data files were converted to NIfTI (Neuroimaging Informatics Technology Initiative) format for use with the DTI-TK software package. Following correction for eddy currents and standard preprocessing (Smith et al., 2004), tensors were reconstructed, registered, and normalized to a study-specific template. NODDI modeling was performed with the microstructure diffusion toolbox (MDT; Harms et al., 2017) on a NVIDIA DGX-1 Deep Learning server (8-V100 GPUs, 32 GB RAM, Dual 20-core Intel Xeon E5-2698 v4 $2.2 \mathrm{GHz}$ CPUs and 512-GB system RAM) to remove run time constraints; analytical pipelines were specifically designed for imaging data collected from fixed ex vivo samples (e.g., using recommended diffusivity assumptions $\mathrm{d} \|=$ $0.6 \times 10^{-3} \mathrm{~mm}^{2} / \mathrm{s}$ and the $\mathrm{d}_{\text {iso }}=2 \times 10^{-3} \mathrm{~mm}^{2} / \mathrm{s}$ and using the "WatsonSHStickTortlsoVIsoDot_B0" fitting model as previously recommended; Zhang et al., 2012). Tract-based spatial statistics (TBSS) were then performed with permutation test results for multiple comparisons and threshold-free cluster enhancement (TFCE; Smith and Nichols, 2009) implemented with FSL's Randomize where voxels were considered significant at the $\alpha<0.05$ level following family-wise error correction. Region of interest (ROI) analysis was conducted to examine specific areas selected a priori for their relevance to clinical psychiatric illness. TBSS and ROI analyses and statistical considerations are detailed in the Statistical analyses, below.

\section{Statistical analyses}

For behavioral analyses, elevated-plus maze time in open arms and entries in open arms, Y-maze alternation percentage, and open field mean velocity, distance traveled, and time spent moving data were subjected to twoway ANOVA with genotype and sex as between-subjects factors. Tukey's HSD post hoc comparison was used to detect differences at the $p<0.05$ level. The behavioral data analyzed meets the assumptions of normality and homogeneity of variances. Total sample size for ANOVA analysis was selected per prior recommended total sample sizes given an a priori effect size $\mathrm{f}=0.5, \alpha$ error probability $=0.05$, and $\beta=0.8$

For TBSS, a processing chain was adapted by replacing the standard TBSS registration (FSL's FNIRT) with the DTI-TK registration routine. The TBSS pipeline was applied using the recommended parameters in FSL. An FA threshold of 0.2 was applied for the creation of the skeleton and a permutation test with $n=252$, corrected for multiple comparisons and TFCE was implemented with FSL's Randomize to compare each of the experimental groups to the control group, with $p<0.05$ as threshold for significance. 

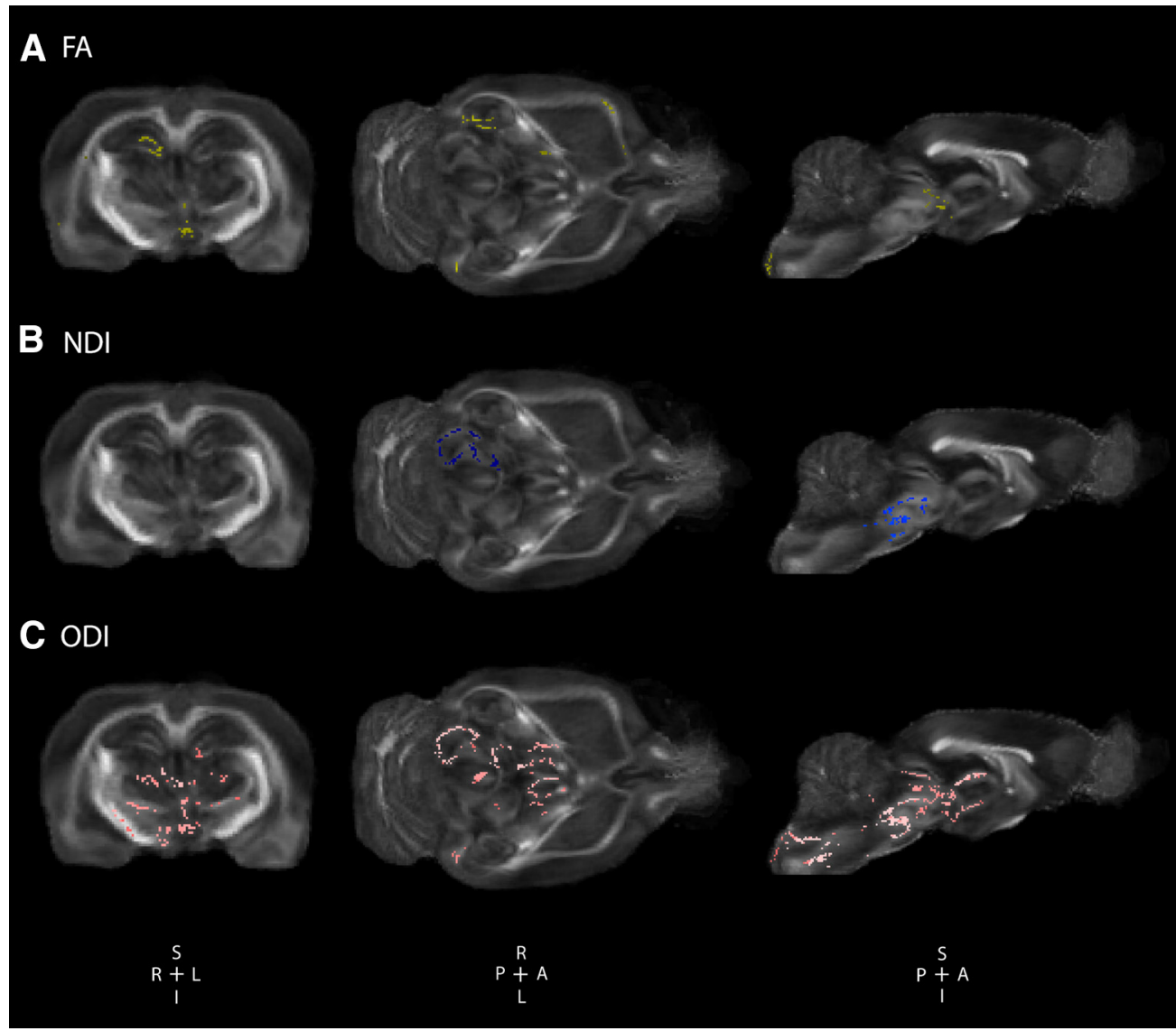

Figure 1. Disc1 sv $\Delta 2$ engenders significant sex-specific global alterations in orientation dispersion in males. Disc1 sv $\Delta 2$ in $\mathrm{P} 120-$ P150 male rats demonstrate deficits in white matter microstructural integrity and contributes to global alterations in neurite density and orientation. $\boldsymbol{A}$, Voxel-wise tract-based spatial statistics significant areas of decreased FA in male Disc1 sv $\Delta 2$ rats $(n=6)$ compared with matched controls ( $n=6$; voxels in yellow). Representative coronal, axial, and sagittal sections reveal significant regions of decreased FA mainly in the right hippocampus, central hypothalamus, and right external capsule. $\boldsymbol{B}$, Disc1 sv $\Delta 2 \mathrm{male}$ rats demonstrated significant areas of decreased NDI compared with matched controls (voxels in blue). Representative coronal, axial, and sagittal sections reveal significant regions of decreased NDI predominantly in right substantia nigra. C, Disc1 sv $\Delta 2$ male rats demonstrated significant areas of decreased ODI compared with matched controls (voxels in pink). Representative coronal, axial, and sagittal sections reveal significant regions of decreased ODI in the left hippocampus, bilateral thalamus, hypothalamus, and substantia nigra.

For ROI imaging analyses, the UNC P72 Rat Atlas was normalized to subject common space and masked with predefined ROls. Diffusion measures for all ROls from the atlas were extracted. Following automated volumetric segmentation of the brain, mean values of both diffusion and neurite indices were computed within six ROls (hippocampus, external capsule, basal ganglia, internal capsule, neocortex, and corpus callosum) in each hemisphere for each individual subject. These ROIs were selected based on their relevance to mental illness for both major white matter and gray matter regions. Two-tailed, two-sample, and unequal variance Student's $t$ test was performed comparing $F A$, axial diffusion ( $A D)$, radial diffusion (RD), mean diffusivity (MD) $[M D=(1 / 3)(T R) ; T R=$ trace diffusivity], NDI, and ODI mean values in Disc1 sv $\Delta 2$ animals against age-sexmatched controls. Raw $p$ values were reported and adjusted $p$ values to control for multiple comparisons were calculated using the Benjamini-Hochberg false discovery rate $(\mathrm{FDR})$ correction $(\mathrm{FDR}=0.05)$. Previous power analyses indicated observed effect size values of $d=2.5$ or greater given low standard deviation between within-group replicates ( $\sigma$ between 0.01 and 0.001), validating sample sizes of six replicates per group. All data are available on reasonable request from the authors.

\section{Results}

\section{Disc1 sv $\Delta 2$ harbors minimal voxel-wise changes in white matter microstructural integrity}

To explore and characterize the influence of early truncation of the major isoform of Disc1 on white matter microstructure, ex vivo DTI was performed. Voxel-wise TBSS analysis of FA was performed comparing the Disc1 $\mathrm{sv} \Delta 2$ model to age and sex-matched controls at a range of postnatal days from P120 to P150. TBSS analysis revealed that Disc1 sv $\Delta 2$ male rats harbor a minimal number of significant voxels of decreased FA when compared with matched controls (Fig. 1A). Similar to the male 

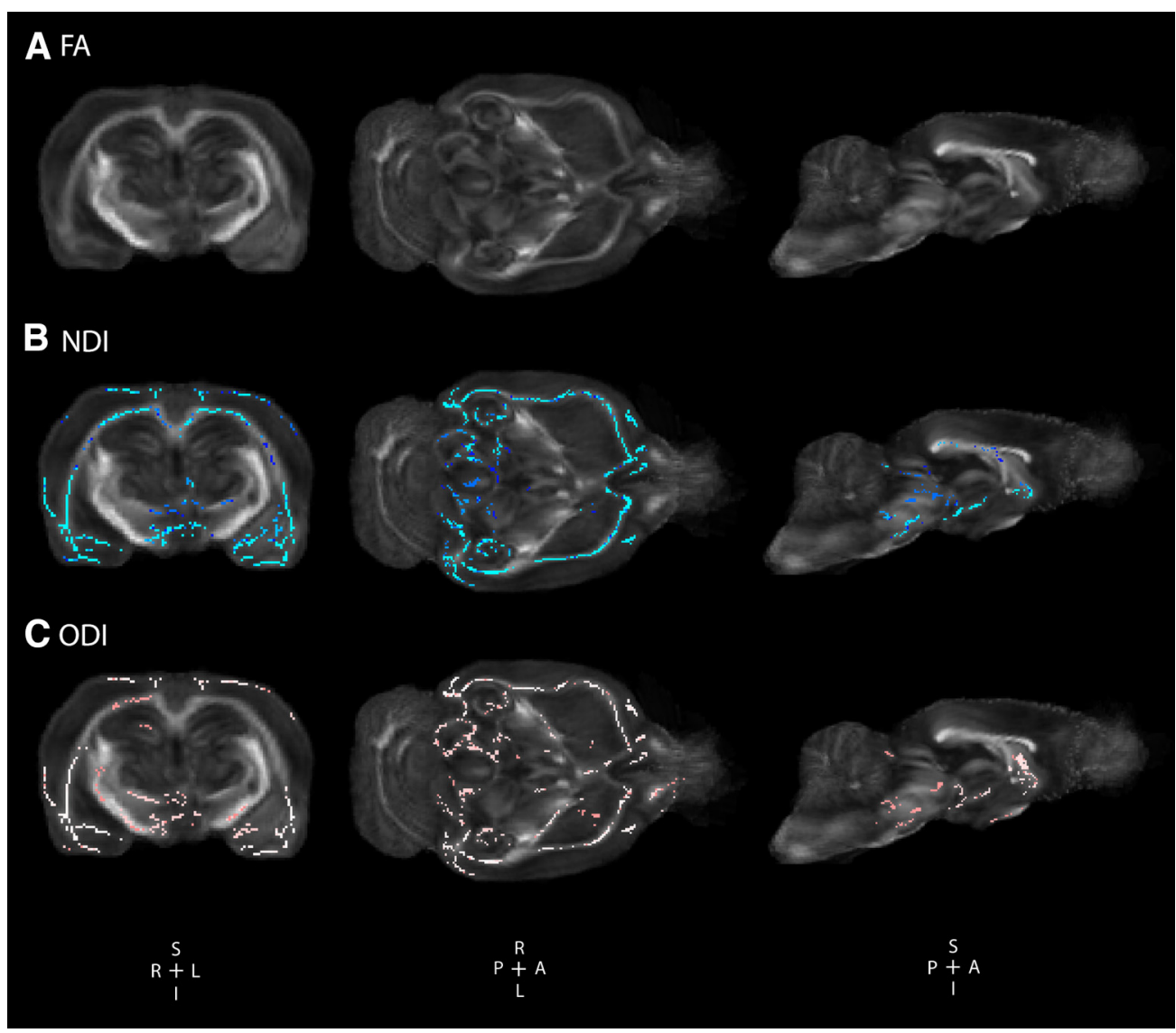

Figure 2. Disc1 sv $\Delta 2$ engenders significant sex-specific global alterations in neurite density and orientation in females. Disc1 sv $\Delta 2$ in P120-P150 female rats demonstrate significant increases in neurite density and orientation dispersion compared with matched controls. A, Voxel-wise TBSS reveal no areas of significant difference in female Disc 1 sv $\Delta 2$ rats $(n=6)$ compared with female controls $(n=6)$. $\boldsymbol{B}$, Disc1 sv $\Delta 2$ female rats demonstrated significant areas of increased NDI compared with female controls (voxels in blue). Representative coronal, axial, and sagittal sections show significant regions of increased NDI in neocortex, external capsule, corpus callosum, basal forebrain, thalamus, and hypothalamus. C, Disc 1 sv $\Delta 2$ female rats demonstrated significant increases in ODI compared with female controls (voxels in pink). Representative coronal, axial, and sagittal sections show significant regions of increased ODI in neocortex, external capsule, internal capsule, corpus callosum, basal forebrain, thalamus, and hypothalamus.

comparison, there were no significant voxel-wise differences in FA between Disc1 sv $\Delta 2$ females and matched wildtype controls (Fig. 2A).

\section{Disc1 sv $\Delta 2$ engenders significant sex-specific global alterations in neurite density and orientation}

To further explore the role of Disc1 on neural structure and organization, ex vivo NODDI was also performed. Voxel-wise TBSS analysis uncovered areas of decreased NDI and ODI values in male Disc1 sv $\Delta 2$ rats when compared with age and sex-matched controls (P120-P150). Decreased NDI values were seen in right substantia nigra and decreased ODI values were observed in left hippocampus and bilateral thalamus, hypothalamus, and substantia nigra (Fig. 1B,C). Disc1 $\mathrm{s} v \Delta 2$ female rats demonstrated significant areas of increased NDI compared with female control rats in bilateral neocortex, external capsule, corpus callosum, basal forebrain, thalamus, and hypothalamus. Disc 1 $\mathrm{s} v \Delta 2$ female rats also demonstrated significant increases in ODI compared with female controls in neocortex, external capsule, internal capsule, corpus callosum, basal forebrain, thalamus, hypothalamus, and right hippocampus (Fig. 2B,C).

\section{Disc1 sv $\Delta 2$ contributes significant sex-specific changes in neural microstructure in regions salient to psychiatric illness}

To a far greater degree than our DTI analysis, NODDI analyses sensitively capture microstructural differences in our Disc 1 sv $\Delta 2$ model across the FA skeleton when compared with age and sex-matched controls. To further explore the impact of early truncation of Disc1 in salient regions of the brain implicated in neuropsychiatric illnesses, a ROI analysis was performed. Six ROls were a priori selected for further analysis: the neocortex, external capsule, corpus callosum, internal capsule, hippocampus, and basal ganglia (including the caudate, putamen, 
Table 1: Disc1 sv $\Delta 2$ contributes to sex-specific significant changes in neural microstructure in salient regions implicated in psychiatric illness

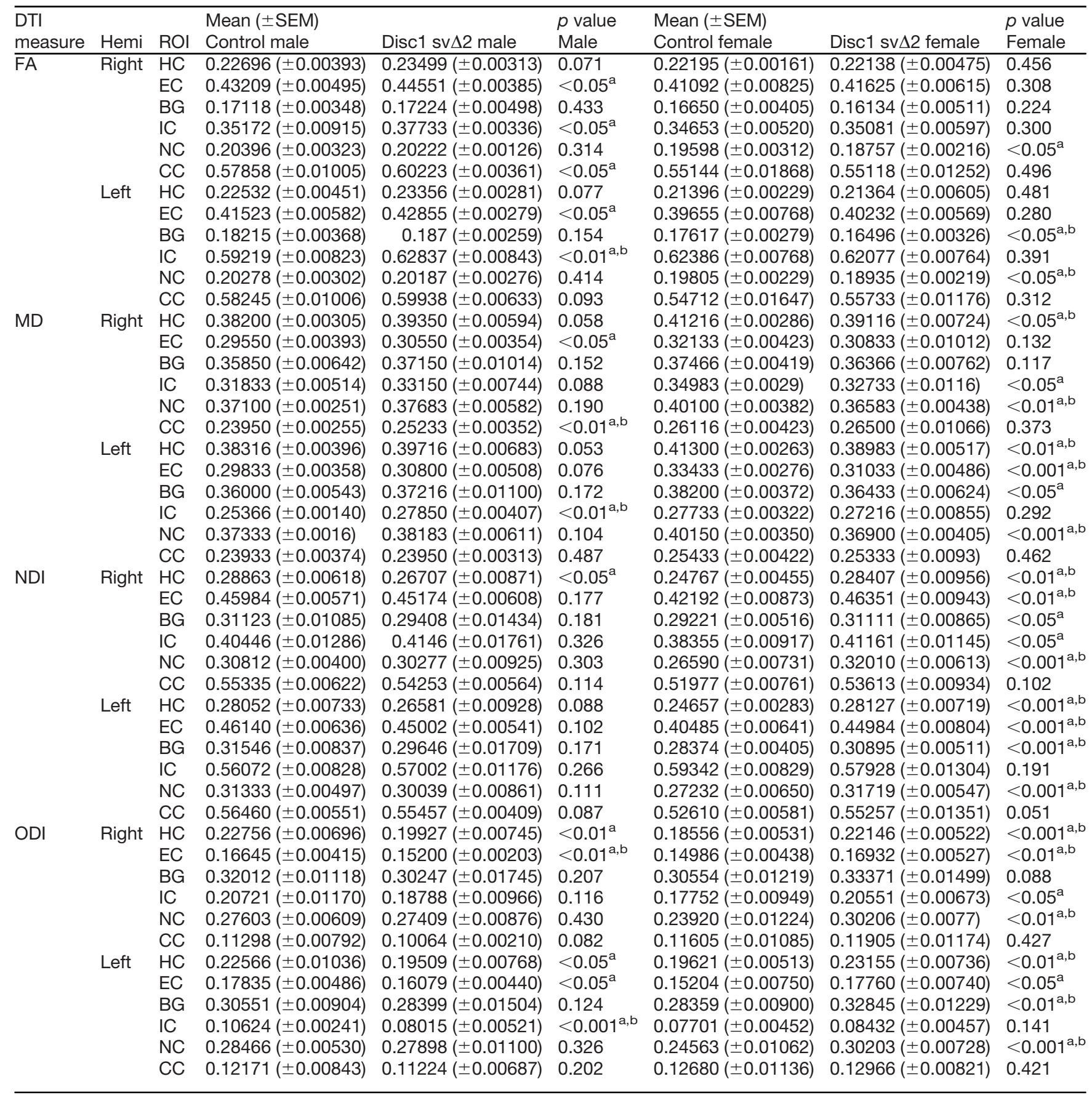

All values are mean \pm SEM. Units of measure for FA, MD, NDI, and ODI are $10^{3} \mathrm{~mm}^{2} / \mathrm{s}$. ROls correspond to ROls derived from the P72 UNC Atlas. Diffusion measure abbreviations: $\mathrm{Hemi}=$ hemisphere; $\mathrm{FA}=$ fractional anisotropy; $\mathrm{MD}=$ mean diffusivity, $\mathrm{NDI}=$ neurite density index; $\mathrm{ODI}=$ orientation dispersion index. $\mathrm{ROI}$ abbreviations: $\mathrm{HC}$ = hippocampus; $\mathrm{EC}=$ external capsule; $\mathrm{BG}=$ basal ganglia; IC = internal capsule; $\mathrm{NC}=$ neocortex; $\mathrm{CC}=$ corpus callosum. For all sample groups, $n=6$.

a Statistically significant.

${ }^{\mathrm{b}}$ Statistically significant after controlling the FDR with the Benjamini-Hochberg procedure $(F D R=0.05)$.

and globus pallidus). Following automated volumetric segmentation of the brain, mean values of both diffusion and neurite indices were computed within each ROI (left and right) for each individual subject for a total of 12 calculated ROls per subject. Disc1 sv $\Delta 2$ male rats harbor significantly increased FA values in the bilateral external capsule, bilateral internal capsule, and right corpus callosum compared with age-matched and sex-matched controls. Additionally, Disc1 sv $\Delta 2$ male rats demonstrated significantly increased MD values in the right external capsule, right corpus callosum, and left internal capsule. Disc1 sv $\Delta 2$ male rats only demonstrated limited 
decreases in NDI, with a significant decrease in the right hippocampus compared with control male rats. Finally, Disc1 sv $\Delta 2$ male rats had significantly reduced ODI in the bilateral hippocampus, bilateral external capsule, and left internal capsule (Table 1). Stricter analyses controlling for multiple comparisons (FDR with the Benjamini-Hochberg procedure with an FDR set to 0.05 ) sustained the significant difference findings for FA in the left internal capsule and ODI in the right external capsule and left internal capsule. Disc1 sv $\Delta 2$ female rats demonstrated significantly decreased FA in the bilateral neocortex and left basal ganglia ROls as well as significantly decreased MD in bilateral hippocampus, left external capsule, left basal ganglia, right internal capsule, and bilateral neocortex. In a reversal of the direction of changes in neural microstructure, significant increases in NDI were observed in bilateral hippocampus, external capsule, basal ganglia, neocortex, as well as right internal capsule. Disc1 sv $\Delta 2$ female rats also had significantly increased ODI in bilateral hippocampus, external capsule, and neocortex, as well as in left basal ganglia and right internal capsule ROIs (Table 1). Of these significant results, the findings for $F A$ in the left basal ganglia and left neocortex, NDI in bilateral hippocampus, external capsule, neocortex, and left basal ganglia, and ODI in bilateral hippocampus, neocortex, right external capsule, and left basal ganglia were significant after applying the FDR procedure.

In addition to the statistical analyses comparing Disc1 sv $\Delta 2$ and control ROls within male and female rats, additional statistical analyses directly comparing Disc1 sv $\Delta 2$ male rats to Disc1 sv $\Delta 2$ female rats found significantly higher FA in male rats and significantly higher $\mathrm{ODI}$ in female rats. Disc1 sv $\Delta 2$ male rats demonstrated significantly higher FA in bilateral hippocampus, external capsule, neocortex, corpus callosum, left basal ganglia, and right internal capsule. Disc1 sv $\Delta 2$ female rats demonstrated significantly higher ODI in bilateral hippocampus, external capsule, right neocortex, and left basal ganglia. There were no significant differences between male Disc1 sv $\Delta 2$ and female Disc1 sv $\Delta 2$ rats for MD or NDI (Table 2).

\section{Disc1 sv $\Delta 2$ behavioral endophenotypes reinforce patterns of sex-specific alteration in neural microstructure}

Disc1 sv $\Delta 2$ male rats exhibited a consistent pattern of behavioral impairments at the P120-P150 time point. We investigated whether Disc1 $\mathrm{sv} \Delta 2$ rats exhibited anxiouslike behavior in the elevated plus maze, which has two opposed closed arms with high walls and two opposed open arms without walls. The duration of time on and frequency of entry into the open arms was significantly lower in Disc1 sv $\Delta 2$ male rats than in wild-type male rats, as well as in Disc $1 \mathrm{sv} \Delta 2$ male rats versus Disc $1 \mathrm{sv} \Delta 2$ female rats (Fig. $3 A, B$ ). We assessed working memory using a free choice three-arm Y-maze. Alternations, consisting of entry into each of the three arms in succession, were recorded as a percentage of the maximum number of alternations possible (alternation percentage). The alternation percentage was significantly lower in Disc1 sv $\Delta 2$ male rats than in wild-type male rats, as well as in Disc1 sv $\Delta 2$ male rats versus Disc1 sv $\Delta 2$ female rats (Fig. $3 C$ ). In a novel, open field, Disc1 sv $\Delta 2$ male rats exhibited significantly higher locomotion, composed of average velocity, distance traveled, and time spent moving, than wild-type male rats, while Disc1 sv $\Delta 2$ female rats exhibited significantly lower time spent moving than wild-type female rats (Fig. 3D-F). That Disc1 sv $\Delta 2$ female rats display significantly lower time spent moving, concomitantly with a statistically similar average velocity and distance traveled compared with wild-type female rats, suggests that these Disc1 sv $\Delta 2$ female rats displayed greater locomotion speed and distance covered throughout the 10-min testing period when they were actually moving.

\section{Discussion}

Here, we demonstrate the ability of NODDI to highlight neurodevelopmental trajectories and differentiate sexspecific changes in brain microstructure that are otherwise difficult to observe with DTI and further corroborate these changes with observed sex-specific differences in systems-level animal behavior. These findings inform the potential application and clinical translational utility of NODDI in studies of brain microstructure in psychiatric illness throughout neurodevelopment and further, the ability of advanced DWI methods such as NODDI to examine the role of biological sex and its influence on brain microstructure in psychiatric illness. Longitudinal or cross-sectional experimental designs incorporating multiple neurodevelopmental timepoints are essential to capture the degree and spatial distribution of neural microstructural change that occurs over different stages of mental illness (Bracht et al., 2015; Herringa, 2017; Seitz et al., 2018). Despite this, longitudinal imaging studies of psychiatric illness across the neurodevelopmental spectrum are relatively rare; however, the few available cross-sectional analyses at different age points have suggested that some DWI methods can identify age-dependent pathologic changes over the course of disease development (Pasternak et al., 2012, 2015). While these studies largely describe neurodevelopmental alterations in measures of DTI and other DWI techniques, the findings presented herein provide insight into the potential for NODDI to sensitively and specifically detect neurodevelopmental changes in brain microstructure beyond those provided by conventional DWI techniques.

The relative advantages provided by NODDI compared with traditional FA analyses is exemplified by the Disc1 sv $\Delta 2$ male neuroimaging findings. Surprisingly, Disc1 $\mathrm{sv} \Delta 2$ male rats at P120-P150 demonstrate a minimal number of significant voxels of decreased FA when compared with matched controls with TBSS analysis. This finding comes in contrast to previously observed global FA decreases in Disc1 sv $\Delta 2$ male rats when compared with matched controls at a P84 time point with TBSS analysis (Barnett et al., 2019). Additionally, on a ROI basis, Disc1 sv $\Delta 2$ male rats did have significantly increased FA values in the bilateral external capsule, bilateral internal capsule, and left corpus callosum compared with agematched and sex-matched controls. These ROI findings 
Table 2: Intersex comparison of Disc1 sv $\Delta 2$ neural microstructure

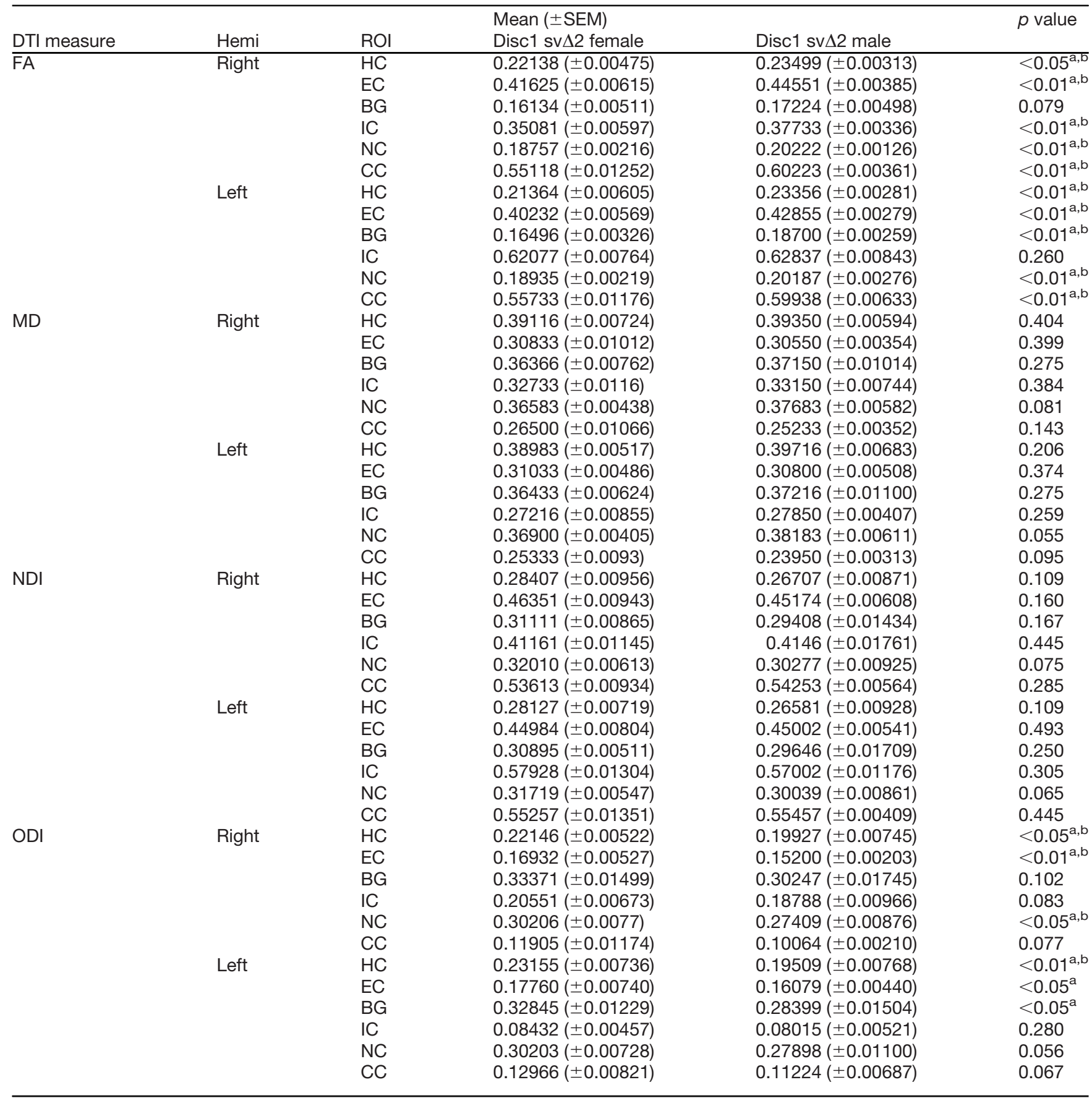

All values are mean \pm SEM. Units of measure for FA, MD, NDI, and ODI are $10^{3} \mathrm{~mm}^{2} / \mathrm{s}$. ROIs correspond to ROIs derived from the P72 UNC Atlas. Diffusion measure abbreviations: Hemi = hemisphere; FA = fractional anisotropy; $\mathrm{MD}=$ mean diffusivity, $\mathrm{NDI}=$ neurite density index; ODI = orientation dispersion index. ROI abbreviations: $\mathrm{HC}=$ hippocampus; $\mathrm{EC}=$ external capsule; $\mathrm{BG}=$ basal ganglia; $\mathrm{IC}=$ internal capsule; $\mathrm{NC}=$ neocortex; $\mathrm{CC}=\mathrm{corpus}$ callosum. For all sample groups, $n=6$.

a Statistically significant.

${ }^{\mathrm{b}}$ Statistically significant after controlling the FDR with the Benjamini-Hochberg procedure $(F D R=0.05)$.

indicate greater potential specificity to FA alteration than can be observed with TBSS voxel-wise analyses, which can partly be attributed to the TBSS technique itself, which considers maximum differences in FA in neighboring voxels in the FA skeleton as opposed to an ROI approach, which accounts for differences in average FA in all voxels in the ROI. The Disc1 sv $\Delta 2$ male ROI findings indicate surprising differences in FA from P84 to P120P150 specifically in major white matter fiber tracts. Prior DTI studies describe FA decreases in frontal commissural and association fiber tracts in human DISC1 $\mathrm{t}(1 ; 11)$ translocation carriers and in DISC1 Ser704Cys SNP allele carriers at adult time points (Sprooten et al., 2011; Whalley et al., 2015). Our findings of increased FA at this time point 
A

Elevated Plus Maze

Open Arm Duration

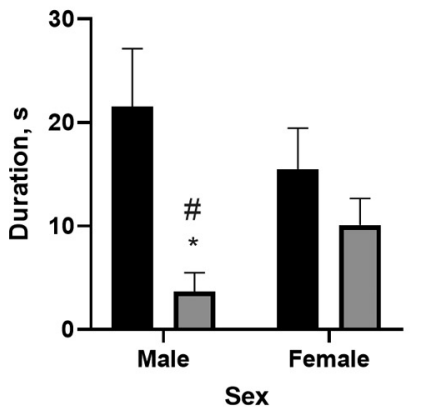

C

\section{Alternation Percentage}

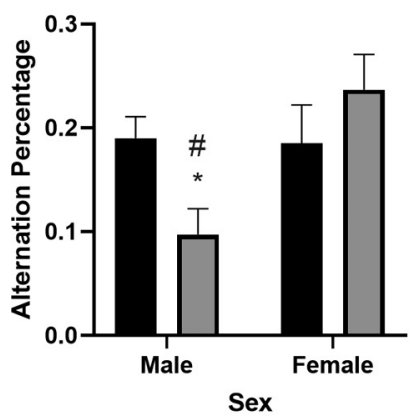

E

Open Field

Distance Moved

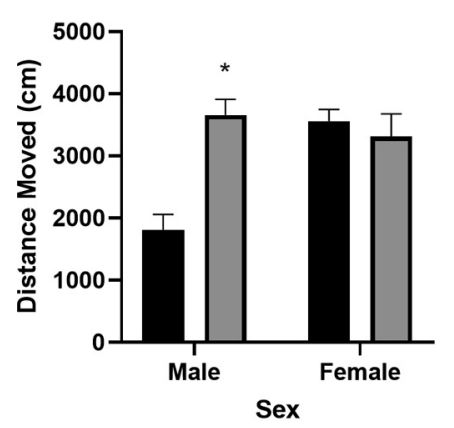

B

Elevated Plus Maze

Open Arm Frequency

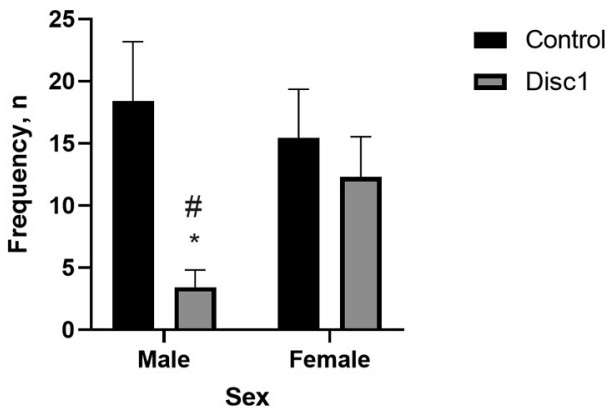

D

$$
\begin{gathered}
\text { Open Field } \\
\text { Average Velocity }
\end{gathered}
$$

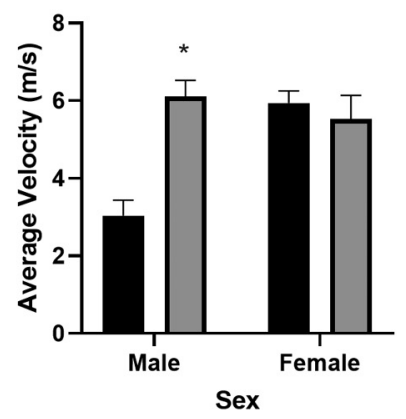

$\mathbf{F}$

Open Field

Time Spent Moving

- Control

口 Disc1

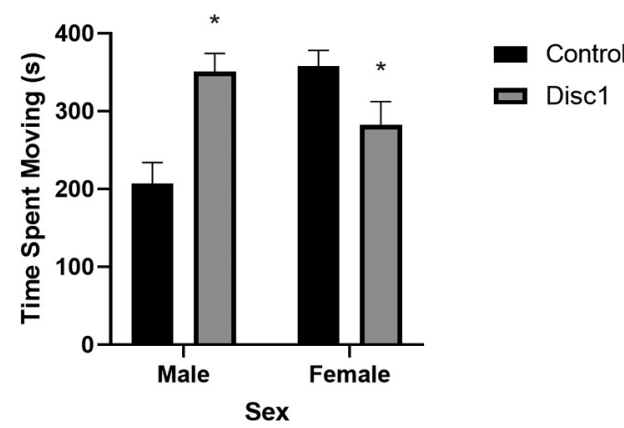

Figure 3. Disc1 sv $\Delta 2$ behavioral endophenotypes reinforce patterns of sex-specific alteration in neural microstructure. $\boldsymbol{A}$, Mean duration in open arms of the elevated-plus maze ( \pm SEM) of wild-type male $(n=12)$, Disc1 sv $\Delta 2$ male $(n=9)$, wild-type female $(n=12)$, and Disc1 sv $\Delta 2$ female $(n=10)$ rats. There was a significant effect of genotype $\left(F_{(1,39)}=8.081, p=0.007\right)$. Mean duration was significantly shorter in Disc1 sv $\Delta 2$ males versus wild-type males $(p=0.007)$, denoted by *. Mean duration was significantly shorter in Disc 1 sv $\Delta 2$ males versus Disc1 sv $\Delta 2$ females $(p=0.032)$, denoted by \#. $\boldsymbol{B}$, Mean frequency in open arms of the elevated-plus maze ( \pm SEM) of wild-type male $(n=12)$, Disc1 sv $\Delta 2$ male $(n=9)$, wild-type female $(n=12)$, and Disc 1 sv $\Delta 2$ female $(n=10)$ rats. There was a significant effect of genotype $\left(F_{(1,39)}=5.476, p=0.025\right)$. Mean frequency was significantly lower in Disc1 sv $\Delta 2$ males versus wildtype males $(p=0.008)$, denoted by *. Mean frequency was significantly lower in Disc1 sv $\Delta 2$ males versus Disc1 sv $\Delta 2$ females $(p=0.013)$, denoted by \#. C. Mean alternation percentage in the y-maze ( \pm SEM) of wild-type male $(n=12), D i s c 1$ sv $\Delta 2$ male $(n=9)$, wild-type female $(n=12)$, and Disc1 sv $\Delta 2$ female $(n=10)$ rats. There was a significant effect of sex $\left(F_{(1,39)}=4.875, p=0.033\right)$ and genotype $\times$ sex interaction $\left(F_{(1,39)}=5.620, p=0.023\right)$. Mean alternation percentage was significantly lower in Disc 1 sv $\Delta 2$ males versus wild-type males $(p=0.005)$, denoted by *. Mean alternation percentage was significantly lower in Disc 1 sv $\Delta 2$ males versus Disc1 $\mathrm{sv} \Delta 2$ females $(p=0.002)$, denoted by \#. $\boldsymbol{D}$, Mean open field velocity ( \pm SEM) of wild-type male $(n=12)$, Disc $1 \mathrm{sv} \Delta 2 \mathrm{male}(n=9)$, wildtype female $(n=12)$, and Disc1 sv $\Delta 2$ female $(n=10)$ rats. There was a significant effect of genotype $\left(F_{(1,39)}=9.125, p=0.004\right)$, sex 
continued

$\left(F_{(1,39)}=7.003, p=0.012\right)$, and genotype $\times$ sex interaction $\left(F_{(1,39)}=15.65, p<0.001\right)$. Mean velocity was significantly higher in Disc1 $\mathrm{sv} \Delta 2$ males versus wild-type males $(p<0.001)$, denoted by *. $\boldsymbol{E}$, Mean open field distance traveled $( \pm$ SEM) of wild-type male $(n=12)$, Disc1 sv $\Delta 2$ male $(n=9)$, wild-type female $(n=12)$, and Disc1 sv $\Delta 2$ female $(n=10)$ rats. There was a significant effect of genotype $\left(F_{(1,39)}=9.134, p=0.004\right)$, $\operatorname{sex}\left(F_{(1,39)}=7.000, p=0.012\right)$, and genotype $\times$ sex interaction $\left(F_{(1,39)}=15.71, p<0.001\right)$. Mean distance traveled was significantly higher in Disc1 sv $\Delta 2$ males versus wild-type males $(p<0.001)$, denoted by *. $\boldsymbol{F}$, Mean open field time spent moving ( \pm SEM) of wild-type male $(n=12)$, Disc1 sv $\Delta 2$ male $(n=9)$, wild-type female $(n=12)$, and Disc1 sv $\Delta 2$ female $(n=10)$ rats. There was a significant genotype $\times$ sex interaction $\left(F_{(1,39)}=18.54, p<0.001\right)$. Mean distance traveled was significantly higher in Disc1 sv $\Delta 2$ males versus wild-type males $(p<0.001)$ and in Disc1 sv $\Delta 2$ females versus wild-type females $(p=0.022)$, denoted by *.

stand as part of a complex axis of findings in regards to changes to FA where prior work in our laboratory and many studies of the human clinical population indicate decreased FA, while other findings present increased FA in schizophrenia patients especially in subcortical white matter (Seok et al., 2007; Rotarska-Jagiela et al., 2009; Alba-Ferrara and de Erausquin, 2013). These findings demonstrate the potential value of assaying multiple timepoints to identify dynamic neurodevelopmental transformations as well as the collection of parameters other than FA to assess other changes in neural microstructure that may also be occurring. The findings of increased FA and $\mathrm{MD}$ in white matter tracts in these ROI analyses in Disc1 sv $\Delta 2$ males may represent a pattern of deficient axonal pruning that has been previously reported in autism spectrum disorder (Valnegri et al., 2017; Suetterlin et al., 2018; Oldehinkel et al., 2019; Pulikkan et al., 2019), while observed decreases to ODI in combination may indicate that these deficiencies may be redundant and inefficient without a normal orientation dispersion of axons. As DISC1 regulates development of synaptic growth and trans-synaptic structure, organization, and function, it would be predicted to impact neuroimaging measures of neurite density and orientation (Camargo et al., 2007; Brandon and Sawa, 2011; Hikida et al., 2012; Furukubo-Tokunaga et al., 2016; Unda et al., 2016). As anticipated, male Disc1 $s v \Delta 2$ rats harbor decreased orientation dispersion across the selected ROls, consistent with previous studies of both dysmorphic and decreased dendritic density and arborization as seen in models of both Disc1 underexpression and overexpression (Miyoshi et al., 2003; Ozeki et al., 2003).

For female animals, at the P120-P150 time point, there were also no significant TBSS differences in FA values between Disc1 sv $\Delta 2$ females and matched wild-type controls. In contrast, $\mathrm{ROI}$ analyses revealed that Disc1 sv $\Delta 2$ female rats demonstrated significantly decreased $F A$ and MD in multiple ROls. The Disc1 sv $\Delta 2$ female rats only demonstrate FA decreases in predominantly gray matter regions at the current time point compared with a more global TBSS and ROI-based observation of decreased FA at the P84 time point. These $\mathrm{ROI}$ findings reiterate the finding of greater specificity to FA decreases that can be observed with $\mathrm{ROI}$ analyses compared with TBSS voxel-wise analyses. Unexpected sex-specific differences were also evident in the distribution of voxel-wise change in measures of NDI and ODI. Disc1 sv $\Delta 2$ female rats demonstrated significantly increased NDI and ODI values (Fig. 2B,C). Previous analyses at P84 in Disc1 sv $\Delta 2$ female rats indicated that NDI was reduced while ODI was not changed at all in comparison to matched controls, and our current results indicate an observed increase in NDI and ODI measures. Previous study of measures of neurite density and orientation dispersion in the context of schizophrenia and first episode psychosis have observed significant decreases in NDI across a range of interhemispheric, corticospinal, and association tracts, but the studies did not analyze sex differences between male and female participants (Nazeri et al., 2017; Rae et al., 2017). These findings of greater and more diffuse increases to NDI and ODI matter microstructural integrity in female Disc1 sv $\Delta 2$ animals compared with Disc 1 sv $\Delta 2$ male animals suggests a structural predisposition to the psychiatric disease state in the latter. The findings comparing previous P84 results to the current study indicate that females harbor decreased neurite density at earlier points in development but then overcompensate beyond wild-type levels with increased neurite density and dispersion of neurite orientation. In contrast, Disc1 sv $\Delta 2$ males may be able to return neurite density to wild-type levels by adulthood but still retain significant deficits (complexity) in neurite orientation. This structural predisposition to the psychiatric disease state dovetails with the clinically observed increased prevalence of male psychopathy.

In addition to these statistical analyses comparing Disc $1 \mathrm{sv} \Delta 2$ and control ROls within male and female rats, additional $\mathrm{ROI}$ statistical analyses directly compared Disc1 sv $\Delta 2$ male rats to Disc1 sv $\Delta 2$ female rats. In concordance with both the above within-male and within-female ROI comparisons and with the existing literature examining general sex differences in the adult human brain, the direct comparison of Disc1 sv $\Delta 2$ male to Disc1 $\mathrm{sv} \Delta 2$ female rats demonstrates that FA values are significantly lower in female rats than in males (Ritchie et al., 2018). Furthermore, ROI statistical analyses comparing Disc $1 \mathrm{sv} \Delta 2$ male rats to Disc $1 \mathrm{sv} \Delta 2$ female rats show that $\mathrm{ODI}$ values are significantly higher in female rats than in males, which aligns strongly with the finding in withinmale and within-female ROI comparisons that Disc1 sv $\Delta 2$ male rats display significantly lower ODI than control males and Disc1 sv $\Delta 2$ female rats display significantly higher ODI values than control females.

With P120-P150 FA and NDI alterations tempered in Disc1 sv $\Delta 2$ male rats and NDI and ODI decreases reversed in Disc1 sv $\Delta 2$ female rats when compared with P84 subjects, the overall constellation of these findings provides additional experimental context for the wide variability in FA findings seen across human DTI studies of mental illness. Variability in study design, population characteristics, imaging modality parameters, and preprocessing 
steps all certainly contribute to the subsequent variability in study results seen in the literature; however, our study indicates that even with the application of highly homogenous cohorts, FA may be an insufficient tool to assess known neural microstructure alterations, especially in psychiatric illness. Given the rich literature of findings regarding reductions to FA in clinical studies of schizophrenia and other mental illnesses, it is clear that FA is a highly sensitive tool to observe gross white matter microstructure alteration, but at the same time is also relatively non-specific. Even if variability in patient study design were eliminated, advanced multicompartment DWI used in combination with FA analyses may be more capable of identifying salient results as demonstrated in our work herein. Multiple imaging sessions within longitudinal studies that can interrogate the dynamics of neural microstructure change over time can also be used to map the underlying pathology and trajectory of these neuropsychiatric disorders.

Previous behavioral analyses of other Disc1 genetic mouse models have demonstrated heterogenous behavioral profiles, with some showing significantly higher locomotion in the open field and anxiety-related behavior in the elevated plus maze, with others finding no significant differences in open field locomotion, Y-maze alternation, or elevated plus-maze anxiety-related behavior (Clapcote et al., 2007; Hikida et al., 2007; Dachtler et al., 2016). One of these murine models used a missense mutation D453G in exon 5 of the mouse Disc1 gene and observed evidence for disruption of GSK3 $\beta$ as well as anxiety-related behavior in Disc1 mice of both sexes in the elevated-plus maze and open field. Estrogen- $\beta$-catenin interactions could potentially mediate this presentation of sexually dimorphic behavioral phenotypes that differ from the finding of deficits only in males in this current analysis. The finding of both sex-specific and endophenotypic categoryspanning behavioral deficits in the Disc1 sv $\Delta 2$ rat model provides greater content validity and justification for using the Disc1 sv $\Delta 2$ model to interrogate the underlying biology of psychiatric illness. These findings of neural microstructure deficits in our Disc1 sv $\Delta 2$ model (global neural microstructure deficits at P84, global neural microstructure deficits and behavioral deficits at P120-P150) align with the currently understood time course of numerous psychiatric illnesses where structural alterations can be seen in the preclinical stages of the disease before the expression of the clinical phenotype.

In summary, this research illustrates the utility and value of NODDI and advanced DWI methods toward identifying and providing novel insights into neurodevelopmental trajectories as well as sex-specific changes in brain microstructure that cannot be differentiated by traditional diffusion tensor and morphometric analyses alone, while corroborating these changes with clinically relevant animal behavior. Sex-specific differences in neural microstructure and behavioral endophenotypes in the Disc1 $s v \Delta 2$ rat model clearly illustrate the greater extent of microstructural change present in male animals and behavioral deficits mirroring the greater male sex-specific incidence of psychiatric illness in patients with alterations to the Disc1 locus. These efforts aid in understanding the degree of genetic susceptibility imparted by Disc 1 to both neural microstructure and behavioral deficits over the course of the neurodevelopmental timeline and the degree to which the environmental milieu can exacerbate or ameliorate the psychiatric disease state. The stark sexspecific differences and the age-related differences in endophenotypes observed in our analysis provides insight for the design of human neuroimaging studies of psychiatric illness and the import and value of NODDI over more traditional morphometric and DTI methods common employed. Considering biological sex as a separate experimental variable and selecting more specific age ranges creates cohort homogeneity that could more clearly delineate and disambiguate neural microstructural components and drivers of mental illness. Additionally, the combined application of more homogenous cohorts with dimensional traits such as NDI and ODI imaging parameters that more clearly correlate with clinical symptoms and behavioral endophenotypes can contribute to more complete neuropsychiatric subtype definitions and improve our capacity to understand disease risk and treatment options in psychiatric illness.

\section{References}

Alba-Ferrara LM, de Erausquin GA (2013) What does anisotropy measure? Insights from increased and decreased anisotropy in selective fiber tracts in schizophrenia. Front Integr Neurosci 7:9.

Barnett BR, Torres-velázquez M, Yi SY, Rowley PA, Sawin EA, Rubinstein CD, Krentz K, Anderson JM, Bakshi VP, Yu JJ (2019) Sex-specific deficits in neurite density and white matter integrity are associated with targeted disruption of exon 2 of the Disc1 gene in the rat. Transl Psychiatry 9:82.

Bracht T, Linden D, Keedwell P (2015) A review of white matter microstructure alterations of pathways of the reward circuit in depression. J Affect Disord 187:45-53.

Brandon NJ, Sawa A (2011) Linking neurodevelopmental and synaptic theories of mental illness through DISC1. Nat Rev Neurosci 12:707-722.

Callicott JH, Straub RE, Pezawas L, Egan MF, Mattay VS, Hariri AR, Verchinski BA, Meyer-Lindenberg A, Balkissoon R, Kolachana B, Goldberg TE, Weinberger DR (2005) Variation in DISC1 affects hippocampal structure and function and increases risk for schizophrenia. Proc Natl Acad Sci USA 102:8627-8632.

Camargo LM, Collura V, Rain J-C, Mizuguchi K, Hermjakob $\mathrm{H}$, Kerrien S, Bonnert TP, Whiting PJ, Brandon NJ (2007) Disrupted in schizophrenia 1 interactome: evidence for the close connectivity of risk genes and a potential synaptic basis for schizophrenia. Mol Psychiatry 12:74-86.

Clapcote SJ, Lipina TV, Millar JK, Mackie S, Christie S, Ogawa F, Lerch JP, Trimble K, Uchiyama M, Sakuraba Y, Kaneda H, Shiroishi T, Houslay MD, Henkelman RM, Sled JG, Gondo Y, Porteous DJ, Roder JJC (2007) Behavioral phenotypes of Disc1 missense mutations in mice. Neuron 54:387-402.

Cross-Disorder Group of the Psychiatric Genomics Consortium, Lee SH, Ripke S, Neale BM, Faraone SV, Purcell SM, Perlis RH, Mowry BJ, Thapar A, Goddard ME, Witte JS, Absher D, Agartz I, Akil H, Amin F, Andreassen OA, Anjorin A, Anney R, Anttila V, Arking DE, et al. (2013) Genetic relationship between five psychiatric disorders estimated from genome-wide SNPs. Nat Genet 45:984-994.

Dachtler J, Elliott C, Rodgers RJ, Baillie GS, Clapcote SJ (2016) Missense mutation in DISC1 C-terminal coiled-coil has GSK3 $\beta$ signaling and sex-dependent behavioral effects in mice. Sci Rep 6:18748.

Furukubo-Tokunaga K, Kurita K, Honjo K, Pandey H, Ando T, Takayama K, Arai Y, Mochizuki H, Ando M, Kamiya A, Sawa A 
(2016) DISC1 causes associative memory and neurodevelopmental defects in fruit flies. Mol Psychiatry 21:1232-1243.

Gandal MJ, Haney JR, Parikshak NN, Leppa V, Ramaswami G, Hartl C, Schork AJ, Appadurai V, Buil A, Werge TM, Liu C, White KP; CommonMind Consortium; PsychENCODE Consortium; iPSYCHBROAD Working Group, Horvath S, Geschwind DH (2018) Shared molecular neuropathology across major psychiatric disorders parallels polygenic overlap. Science 359:693-697.

Gold AL, Brotman MA, Adleman NE, Lever SN, Steuber ER, Fromm SJ, Mueller SC, Pine DS, Leibenluft E (2016) Comparing brain morphometry across multiple childhood psychiatric disorders. J Am Acad Child Adolesc Psychiatry 55:1027-1037.e3.

Hamshere ML, Bennett P, Williams N, Segurado R, Cardno A, Norton $\mathrm{N}$, Lambert D, Williams H, Kirov G, Corvin A, Holmans P, Jones L, Jones I, Gill M, O'Donovan MC, Owen MJ, Craddock N (2005) Genomewide linkage scan in schizoaffective disorder: significant evidence for linkage at 1q42 close to DISC1, and suggestive evidence at 22q11 and 19p13. Arch Gen Psychiatry 62:1081-1088.

Harms RL, Fritz FJ, Tobisch A, Goebel R, Roebroeck A (2017) Robust and fast nonlinear optimization of diffusion MRI microstructure models. Neuroimage 155:82-96.

Hashimoto R, Numakawa T, Ohnishi T, Kumamaru E, Yagasaki Y, Ishimoto T, Mori T, Nemoto K, Adachi N, Izumi A, Chiba S, Noguchi H, Suzuki T, Iwata N, Ozaki N, Taguchi T, Kamiya A, Kosuga A, Tatsumi M, Kamijima K, et al. (2006) Impact of the DISC1 Ser704Cys polymorphism on risk for major depression, brain morphology and ERK signaling. Hum Mol Genet 15:30243033.

Herringa RJ (2017) Trauma, PTSD, and the Developing Brain. Curr Psychiatry Rep 19:69.

Hikida T, Jaaro-Peled H, Seshadri S, Oishi K, Hookway C, Kong S, Wu D, Xue R, Andradé M, Tankou S, Mori S, Gallagher M, Ishizuka K, Pletnikov M, Kida S, Sawa A (2007) Dominant-negative DISC1 transgenic mice display schizophrenia-associated phenotypes detected by measures translatable to humans. Proc Natl Acad Sci USA 104:14501-14506.

Hikida T, Gamo NJ, Sawa A (2012) DISC1 as a therapeutic target for mental illnesses. Expert Opin Ther Targets 16:1151-1160.

Hodgkinson CA, Goldman D, Jaeger J, Persaud S, Kane JM, Lipsky $\mathrm{RH}$, Malhotra AK (2004) Disrupted in schizophrenia 1 (DISC1): association with schizophrenia, schizoaffective disorder, and bipolar disorder. Am J Hum Genet 75:862-872.

Kilpinen H, Ylisaukko-oja T, Hennah W, Palo OM, Varilo T, Vanhala $R$, Nieminen-von Wendt $T$, von Wendt L, Paunio $T$, Peltonen $L$ (2008) Association of DISC1 with autism and Asperger syndrome. Mol Psychiatry 13:187-196.

Miyoshi K, Honda A, Baba K, Taniguchi M, Oono K, Fujita T, Kuroda S, Katayama T, Tohyama M (2003) Disrupted-in-schizophrenia 1, a candidate gene for schizophrenia, participates in neurite outgrowth. Mol Psychiatry 8:685-694.

Nazeri A, Mulsant BH, Rajji TK, Levesque ML, Pipitone J, Stefanik L, Shahab S, Roostaei T, Wheeler AL, Chavez S, Voineskos AN (2017) Gray matter neuritic microstructure deficits in schizophrenia and bipolar disorder. Biol Psychiatry 82:726-736.

Oldehinkel M, Mennes M, Marquand A, Charman T, Tillmann J, Ecker C, Dell'Acqua F, Brandeis D, Banaschewski T, Baumeister S, Moessnang C, Baron-Cohen S, Holt R, Bölte S, Durston S, Kundu P, Lombardo MV, Spooren W, Loth E, Murphy DGM, et al. (2019) Altered connectivity between cerebellum, visual, and sensory-motor networks in autism spectrum disorder: results from the EU-AIMS longitudinal European autism project. Biol psychiatry Cogn Neurosci neuroimaging 4:260-270.

Ozeki Y, Tomoda T, Kleiderlein J, Kamiya A, Bord L, Fujii K, Okawa M, Yamada N, Hatten ME, Snyder SH, Ross CA, Sawa A (2003) Disrupted-in-schizophrenia-1 (DISC-1): mutant truncation prevents binding to NudE-like (NUDEL) and inhibits neurite outgrowth. Proc Natl Acad Sci USA 100:289-294.

Pasternak O, Westin CF, Bouix S, Seidman LJ, Goldstein JM, Woo TU, Petryshen TL, Mesholam-Gately RI, McCarley RW, Kikinis R, Shenton ME, Kubicki M (2012) Excessive extracellular volume reveals a neurodegenerative pattern in schizophrenia onset. $\mathrm{J}$ Neurosci 32:17365-17372.

Pasternak O, Westin CF, Dahlben B, Bouix S, Kubicki M (2015) The extent of diffusion MRI markers of neuroinflammation and white matter deterioration in chronic schizophrenia. Schizophr Res 1:113-118.

Pulikkan J, Mazumder A, Grace T (2019) Role of the gut microbiome in autism spectrum disorders. Adv Exp Med Biol 1118:253-269.

Rae CL, Davies G, Garfinkel SN, Gabel MC, Dowell NG, Cercignani M, Seth AK, Greenwood KE, Medford N, Critchley HD (2017) Deficits in neurite density underlie white matter structure abnormalities in first-episode psychosis. Biol Psychiatry 82:716-725.

Ritchie SJ, Cox SR, Shen X, Lombardo MV, Reus LM, Alloza C, Harris MA, Alderson HL, Hunter S, Neilson E, Liewald DCM, Auyeung B, Whalley HC, Lawrie SM, Gale CR, Bastin ME, McIntosh AM, Deary IJ (2018) Sex differences in the adult human brain: evidence from 5216 UK biobank participants. Cereb Cortex 28:2959-2975.

Rotarska-Jagiela A, Oertel-Knoechel V, DeMartino F, van de Ven V, Formisano E, Roebroeck A, Rami A, Schoenmeyer R, Haenschel C, Hendler T, Maurer K, Vogeley K, Linden DEJ (2009) Anatomical brain connectivity and positive symptoms of schizophrenia: a diffusion tensor imaging study. Psychiatry Res 174:9-16.

Seitz J, Rathi Y, Lyall A, Pasternak O, del Re EC, Niznikiewicz M, Nestor P, Seidman LJ, Petryshen TL, Mesholam-Gately RI, Wojcik J, McCarley RW, Shenton ME, Koerte IK, Kubicki M (2018) Alteration of gray matter microstructure in schizophrenia. Brain Imaging Behav 12:54-63.

Seok JH, Park HJ, Chun JW, Lee SK, Cho HS, Kwon JS, Kim JJ (2007) White matter abnormalities associated with auditory hallucinations in schizophrenia: a combined study of voxel-based analyses of diffusion tensor imaging and structural magnetic resonance imaging. Psychiatry Res 156:93-104.

Smith SM, Nichols TE (2009) Threshold-free cluster enhancement: addressing problems of smoothing, threshold dependence and localisation in cluster inference. Neuroimage 44:83-98.

Smith SM, Jenkinson M, Woolrich MW, Beckmann CF, Behrens TEJ, Johansen-Berg H, Bannister PR, De Luca M, Drobnjak I, Flitney DE, Niazy RK, Saunders J, Vickers J, Zhang Y, De Stefano N, Brady JM, Matthews PM (2004) Advances in functional and structural MR image analysis and implementation as FSL. Neuroimage 23:S208-S219.

Sprooten E, Sussmann JE, Moorhead TW, Whalley HC, ffrenchConstant C, Blumberg HP, Bastin ME, Hall J, Lawrie SM, McIntosh AM (2011) Association of white matter integrity with genetic variation in an exonic DISC1 SNP. Mol Psychiatry 16:688-689.

Suetterlin P, Hurley S, Mohan C, Riegman KLH, Pagani M, Caruso A, Ellegood J, Galbusera A, Crespo-Enriquez I, Michetti C, Yee Y, Ellingford $R$, Brock $O$, Delogu A, Francis-West $P$, Lerch JP, Scattoni ML, Gozzi A, Fernandes C, Basson MA (2018) Altered neocortical gene expression, brain overgrowth and functional over-connectivity in chd8 haploinsufficient mice. Cereb Cortex 28:2192-2206.

Unda BK, Kwan V, Singh KK (2016) Neuregulin-1 regulates cortical inhibitory neuron dendrite and synapse growth through DISC1. Neural Plast 2016:7694385.

Valnegri P, Huang J, Yamada T, Yang Y, Mejia LA, Cho HY, Oldenborg A, Bonni A (2017) RNF8/UBC13 ubiquitin signaling suppresses synapse formation in the mammalian brain. Nat Commun 8:1271.

Whalley HC, Dimitrova R, Sprooten E, Dauvermann MR, Romaniuk L, Duff B, Watson AR, Moorhead B, Bastin M, Semple SI, Giles S, Hall J, Thomson P, Roberts N, Hughes ZA, Brandon NJ, Dunlop J, Whitcher B, Blackwood DHR, McIntosh AM, et al. (2015) Effects of a balanced translocation between chromosomes 1 and 11 disrupting the DISC1 locus on white matter integrity. PLoS One 10: e0130900.

Zhang H, Schneider T, Wheeler-Kingshott CA, Alexander DC (2012) NODDI: practical in vivo neurite orientation dispersion and density imaging of the human brain. Neuroimage 61:1000-1016. 